\title{
A stochastic simulation model for Asian elephant Elephas maximus populations and the inheritance of tusks
}

\author{
Ralph TIEDEMANN and Fred KURT
}

Tiedemann R. and Kurt F. 1995. A stochastic simulation model for Asian elephant Elephas maximus populations and the inheritance of tusks. [In: Ecological genetics in mammals II. G. B. Hartl and J. Markowski, eds]. Acta Theriologica, Suppl. 3: 111-124.

In the framework of available historical data on population size and human impact on the Asian elephant Elephas maximus Linnaeus, 1758, we developed a stochastic simulation model for elephant populations, which simulates individual elephants and includes their geno- and phenotype regarding the tusk character. The model omits density dependence of reproduction parameters and mortality rates. The model predicts female mortality and mean calving interval to be the paramount factors determining the long-term population trend. For simulated female mortality rates of $5 \%$ and $5.5 \%$ a mean calving interval of 5.2 years and $4.4-4.5$ years, respectively, leads to stable populations, which is in accordance with values derived from field observations. Taking into account a higher mortality of tuskers due to human impact, frequencies of the tusk allele were simulated both for dominant and recessive inheritance of the tusk character. The best accordance with census data was achieved, when the tusk allele was assumed to be dominant, combined with a slight reproductive advantage for tuskers. Since the model simulates population dynamics and tusk allele frequencies under various mortality schedules, it can be used to predict the effect of conservation efforts on populations of the Asian elephant.

Institut für Haustierkunde, Christian-Albrechts-Universität zu Kiel, Olshausenstraße 40, D-24118 Kiel, Germany

Key words: Elephas maximus, population dynamics, selective hunting, secondary sexual character

\section{Introduction}

In some populations of the Asian elephant Elephas maximus Linnaeus, 1758 population sizes have been fairly well documented for a period of about 2500 years (Digby 1971, Trautmann 1982). Within this period, human impact on elephant populations, ie capturing or hunting, was characterized by considerable temporal and local variation (Sukumar 1989, Kurt 1992). This has led to various mortality schedules, which often also differed between sexes and, in some periods, were biased towards tusk bearing males (cf Kurt et al. 1995). This framework provides an unique opportunity for population simulation studies: Population dynamics can be simulated by stochastic models under differing regimes of relevant parameters, such as mean mortality, mean calving interval or mean age at first reproduction. Simulations may then be validated by comparing them with available data about historical population dynamics. 
While population dynamics of the African elephant Loxodonta africana has been modelled using both deterministic and stochastic approaches (Fowler and Smith 1973, Hanks and McIntosh 1973, Wu and Botkin 1980), in Asian elephant the deterministic model of Sukumar (1989) is the only simulation available so far. This simulation covers only a period of 50 years, but already shows the extreme influence of different mortality schedules on population size. In a more recent stochastic model, Sukumar (1992) investigates only survival possibilities of elephant populations according to the initial population size under a regime of stochastic alterations in mortality and birth-rate.

In contrast to all these approaches, our model mainly aims at simulating long-term population dynamics under mortality regimes changing with historical periods. The model also takes into account the inheritance of the tusk character, which is assumed to be controlled by a single autosomal gene or a major gene in a polygene system. Thus, it enables us to investigate the effect of selective hunting for tuskers on the frequency of tusk-bearing males in a population and may give us an idea of the inheritance of tusks (Kurt et al. 1995).

\section{Assumptions of the model}

\section{Lack of migration}

The simulated elephant population is assumed to be reproductively isolated from other populations, ie neither immigration nor emigration is supposed to occur. Each individual enters the population by birth and leaves it by death. Even though some exchange between adjacent populations may occur in reality, this does not need to be included in this model unless it would cause considerable changes in sex ratio, population size, age structure, or in the frequency of tuskers. One rationale behind the assumption of lacking migration is the known philopatry of Asian elephants, as has been shown by several dislocation experiments in Sri Lanka, southern India and Sumatra, where the elephants immediately returned to their places of origin (eg Santiapillai and Suprahman 1985, Jayewardene 1994).

\section{Lack of density dependence}

In our simulation model, we excluded any kind of density dependence in population dynamics. Unlike Fowler and Smith (1973), we do not assume the population to be necessarily limited by the carrying capacity of the environment. In fact, we suppose that real population sizes are below carrying capacity. Thus, both mortality and reproduction parameters are not significantly influenced by population size. We are aware of the assumption of density independence in reproduction and mortality not to hold without limits, if the population would grow infinitely. However, the growing abilities of elephant populations are limited due to late puberty and long calving intervals (see Hanks and McIntosh 1973), and may even be counteracted by natural mortality, which could lead to fairly stable population sizes below the carrying capacity of the environment. Unlike the 
African elephant, there are no records on an over-exploitation of habitat by Asian elephants, except for a few areas, where elephant populations are isolated in forests overgrazed by cattle (Sivaganesan and Sathyanarayana 1993).

\section{Invariance in reproduction parameters}

Reproduction parameters are specified once for each simulation procedure and are invariant for a single simulation. These parameters are (values which have been used in our simulations are given in parentheses) :

- male age at first reproduction $\left(\mathrm{m}_{0}=20\right.$ years; Kurt 1992, Kurt et al. 1995),

- female age at first reproduction $\left(f_{0}=8\right.$ years; Kurt 1970, 1974),

- gestation period ( $\mathrm{g}=2$ years; eg Sukumar 1989),

- minimum time between two consecutive conceptions for a female $\left(\Delta_{0}=4\right.$ years; Kurt 1970, Sukumar 1989), which corresponds to the minimum calving interval, - sex ratio of offspring at the time of conception (1:1),

- maximum number of females successfully mated annually by one male (6; F. Kurt, in prep.).

These parameters on reproduction are supposed not to be influenced by population size or human impact on the population. Both sexes are considered to be active in reproduction until their death. In Asian elephants even females older than 60 years have been reported to reproduce (Toke Gale 1974, Nair et al. 1992). Moreover, Hanks and McIntosh (1973) showed that the exclusion of the oldest females from reproduction is of little significance for population dynamics.

In general, random mating is assumed between all adults in reproduction. Despite this, sexual selection in favour of tuskers can be included in the simulation. If sexual selection is included, it is considered to be independent of density and mortality.

\section{Short-term invariance in the mean time between two conceptions and in mortality}

The simulation model assumes that the period to be simulated can be divided into time spans in a way that mean time between two conceptions $(\Delta)$ and mean mortalities $\left(\alpha_{\mathrm{f}}, \alpha_{\mathrm{m}}, \alpha_{\mathrm{t}}=\right.$ mortalities of females, maknas and tuskers, respectively) are constant within each time span. Obviously, the neglection of annual variation in these parameters is an artificial simplification of the real situation. For example, availability of resources and hunting effort may vary between years, leading to varying birth- and mortality rates (cf McKay 1973). Nevertheless, since these variations will mainly influence annual fluctuations in population size rather than long-term population dynamics, they need not be considered for the purpose of this simulation.

For a particular mean time between two conceptions $(\Delta)$ to be simulated, we determine a corresponding birth-rate $(\beta)$ for the shortest possible interval between two conceptions $\left(\Delta_{0}\right)$ which fits the following equation:

$$
\Delta=\sum_{i=0}^{\infty} \beta(1-\beta)^{i} \Delta_{i}
$$

where $\Delta_{i}=\left(\Delta_{0}+i\right)$ are subsequent years of the minimum number of years between two conceptions. As an example, for values of $\Delta=4.43$ years and $\Delta_{0}=4$ years, the 
corresponding birth-rate is $\beta=0.7$. This means that on average $70 \%$ of the female elephants are assumed to become pregnant in the 4th year after a previous conception, $21 \%$ in the 5 th year and the remaining $9 \%$ in the 6 th year or later. Note, that the value of $\Delta$ is identical to the mean calving interval, a parameter estimated by other authors (Kurt 1970, Sukumar 1989).

Two phenotypes of male elephants

In Asian elephant, tusks are restricted to males (eg Evans 1910). In the model, tusks are assumed to be a binary character, ie to be either present or absent (cf Kurt et al. 1995). Tuskless elephants of both sexes lack these incisors or possess so called tushes, which are decurved, thin and generally not longer than $30 \mathrm{~cm}$ (McKay 1973). In the model, tuskless elephants are pooled, regardless of the presence or absence of these tushes. For assessing the mode of inheritance, we suppose that the occurence of tusks is controlled by a single gene or a major gene in a polygene system. In the simulation, the tusk allele is assumed to be either dominant or recessive. The tusk allele and the makna allele are assigned " $A$ " and " $a$ ", respectively. The dominance status of the tusk allele is specified prior to the simulation and is invariant for each particular simulation run.

\section{The concept of the population simulation}

\section{The concept of stochasticity}

In the population model, each single process whose outcomes can be described in probabilities, is simulated stochastically: Whether a particular elephant dies, becomes pregnant or - in the case of being heterozygous - inherits an " $A$ " or " $a$ " allele, is always the result of a random procedure. Thus, the outcome of the simulation is not predictable. Instead, the simulation has to be repeated several times with the same parameters to get an idea about the influence of random effects on population dynamics.

\section{The concept of individual elephants}

Similar to the "individual life history approach" in the Loxodonta africana population simulation of $\mathrm{Wu}$ and Botkin (1980), the simulated population consists of individual elephants. Each male is characterized by the tupel (age, genotype). Genotype refers to the tusk allele, where $A A$ is a homozygous tusker, $a a$ a homozygous makna, and $A a$ a heterozygous individual, whose phenotype depends on the simulated dominance mode. Each female is characterized by the tripel (age, genotype, delay). Delay is set to $\Delta_{0}$, the minimum time between two conceptions, in case of a fertilization, and a delay $>0$ indicates that this particular female cannot be fertilized due to actual gravidity or lactation. Age and genotype of females are defined as in males, but all females are phenotypically tuskless. At any time, the individual life history approach allows inspection of age structure, 
allele frequencies and deviations from Hardy-Weinberg-expectations with respect to the tusk genotypes.

\section{The initial generation of a population}

Prior to simulation, the starting values for the total number of individuals in both sexes and the initial frequency of the tusk allele $(p)$ are specified. The initial population is in Hardy-Weinberg-equilibrium with regard to the tusk allele and consists of individuals with a random age distribution up to a treshold age, which has to be specified in the beginning. To overcome this unrealistic intial situation and to establish a realistic age distribution, the simulation is allowed to equilibrate for 100 simulated years using a standard set of parameters for the annual birth-rate $(\beta=0.7)$ and the annual mortality ( $\left.\alpha_{\mathrm{f}}=0.055, \alpha_{\mathrm{m}}=\alpha_{\mathrm{t}}=0.07\right)$, the latter being similar to the "low mortality schedule" used in the short-term population model of Sukumar (1989). The birth-rate corresponds to a mean calving interval of $\Delta=4.43$.

\section{The simulation of one year}

The smallest time unit in the population model is one year. A simulated year starts with the stochastic mortality simulation for the males (Fig. 1). Depending on whether a particular male is phenotypically a tusker or a makna, its probability to die is $\alpha_{t}$ or $\alpha_{m}$. If the male lives and has reached the age of first reproduction $\left(\mathrm{m}_{0}\right)$, it contributes to the percentage of its particular genotype ( $p_{A A}, p_{A a}$ or $p_{a a}$, respectively).

Next, the female mortality is simulated (Fig. 2). If a particular female lives and is not in a delay status (ie actual gravidity or lactation), it has a probability of $\beta$, the birth-rate, to get pregnant, unless the number of females already fertilized that year exceeds 6 per reproductive male. In the latter case, the female is assumed not to reproduce that year.

If a female does reproduce, the genotype of the offspring is determined as shown in Fig. 2. Note, that the simulation does not choose a particular male for mating, but rather takes into account the probabilities to mate with a male of a particular genotype.

\section{The simulation of sexual selection}

The model includes the possibility to simulate sexual selection in favour of or against tuskers. For the purpose of the simulation, it does not matter whether this selective advantage might be due to female choice or due to the ability of the male to monopolize on average more females. Actually, the latter has never been observed in elephants, neither in Loxodonta nor in Elephas (cf Poole 1987, 1989). In the model, the selection coefficient for tuskers $(s)$ is specified in relation to that in the maknas, ie $s=1.5$ means $50 \%$ selective advantage for tuskers. When simulating sexual selection, the probabilities for the reproduction of the different male genotypes are recalculated as follows: 


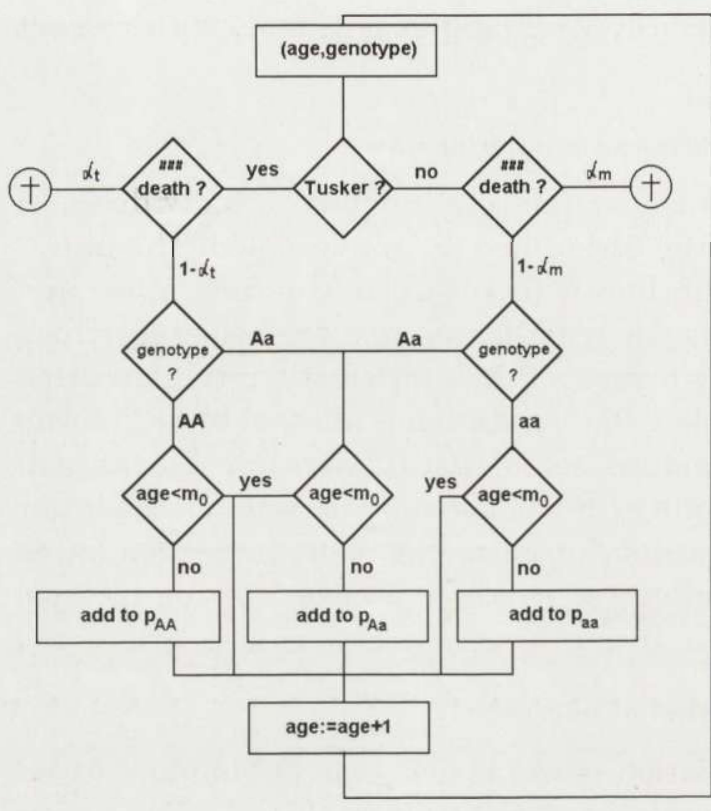

Fig. 1. Scheme of the simulation of one year for one male elephant, characterized by its age and its genotype. \#\#\# marks a random process. The respective probabilities are given along the subsequent lines.

$$
\begin{aligned}
& p_{A A}=s^{*} N_{A A} /\left[N_{\text {makna }}+\left(s^{*} N_{\text {tusker }}\right)\right] \\
& p_{a a}=N_{a a} /\left[N_{\text {makna }}+\left(s^{*} N_{\text {tusker }}\right)\right], \\
& p_{A a}=s^{*} N_{A a} /\left[N_{\text {makna }}+\left(s^{*} N_{\text {tusker }}\right)\right],
\end{aligned}
$$

in case of tusker dominance and

$p_{A a}=N_{A a} /\left[N_{\text {makna }}+\left(s^{*} N_{\text {tusker }}\right)\right]$,

in case of makna dominance.

$N_{A A}, N_{A a}, N_{a a}, N_{\text {makna }}$ and $N_{\text {tusker }}$ are the absolute numbers of reproductive males of the specified genotype and phenotype, respectively.

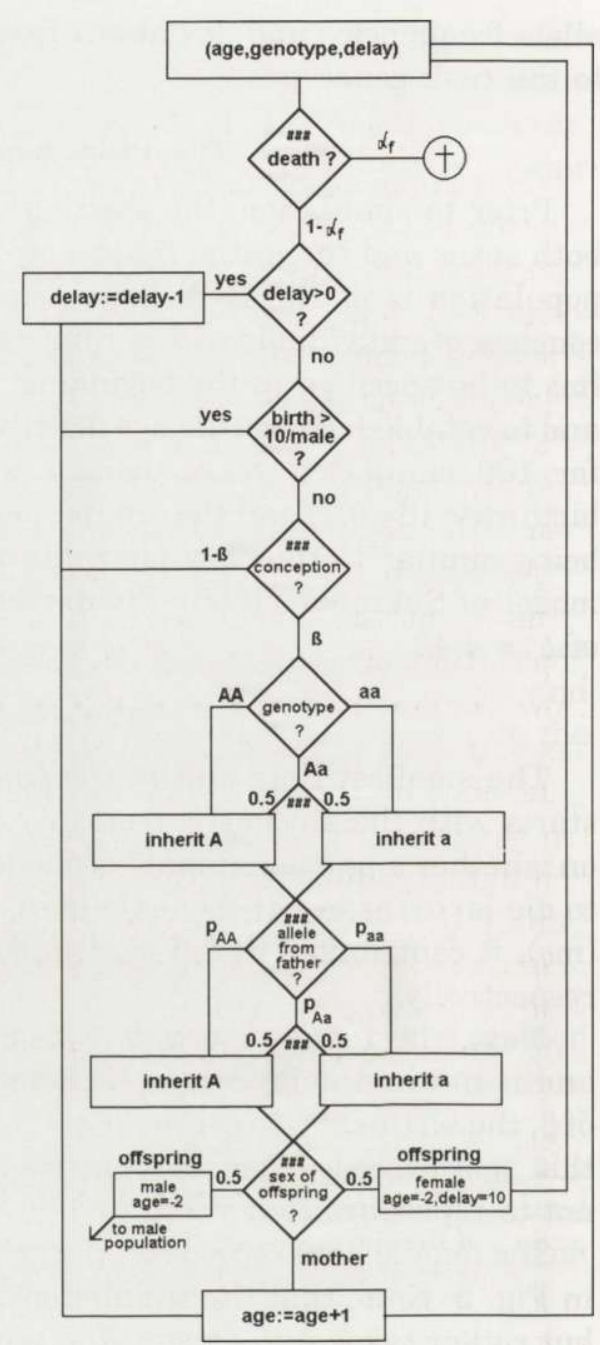

Fig. 2. Scheme of the simulation of one year for one female elephant with the possibility of reproduction. \#\#\# is used as in Fig. 1. 


\section{Application of the model}

\section{Influence of calving interval and mortality on population dynamics}

One application of the model is the investigation of population dynamics in relation to different schedules of birth- and mortality rates, regardless of the tusk character. For this purpose, we generated a population of 450 elephants. This is about 3-4 times the size of the largest known social clan of wild-living elephants. The idea behind this is that adult bulls migrate - if at all - only between $2-3$ adjacent clans (Kurt 1974, A. Desai, pers. comm.). The initial population consisted of 300 females and 150 males, which is a realistic sex ratio for populations with medium male mortality (eg Sukumar 1989). After an equilibration time of 100 years, the population was simulated for 500 years.

As may be expected for a polygynous species like the elephant, the alteration of male mortality mainly influences the sex ratio, but has little effect on total population size, unless a drastic mortality schedule is simulated (Table 1). However, in the latter case the population may become extinct shortly after the sex ratio has reached a critical value skewed towards females, which - of course - depends on the maximum number of females annually mated by a single male. On the contrary, a small change in female mortality has dramatic effects on the simulated population (Table 1). Actually, if we assume the population not to be limited by the carrying capacity of the environment, the specific relation between mean calving interval $(\Delta)$ and female mortality $\left(\mathrm{m}_{\mathrm{f}}\right)$ causes the population to be stable, growing or extinct (Fig. 3). For a given female mortality, only a narrow range of values for the calving interval leads to a stable population. For the

Table 1. Changes in population size and sex ratio under different mortality schedules $(\Delta=4.43$, initial population size $=450$, initial female:male ratio $2: 1,500$ years simulated). $\mathrm{m}_{\mathrm{f}}-$ female mortality, $\mathrm{m}_{\mathrm{t}}$ - tusker mortality, $\mathrm{m}_{\mathrm{m}}$ - makna mortality (in \%/year), $\mathrm{N}$ - number of simulation runs, $\check{\mathrm{r}}$ - overall median of the simulation medians of percent change in population size per year, $Q_{1} \breve{r}, Q_{2} \check{r}-25 \%$ and $75 \%$ quartils of the simulation medians of percent change in population size per year, $\breve{s}-$ overall median of the simulation medians of sex ratio skewed towards females (* indicates the absence of reproductive males in more than half of the simulated years), Q1š, Q 25 - $25 \%$ and $75 \%$ quartils of the simulation medians of sex ratio skewed towards females, Y - median of time until extinction in simulations where the population did not survive.

\begin{tabular}{rrrrrrrrrrr}
\hline $\mathrm{m}_{\mathrm{f}}$ & $\mathrm{m}_{\mathrm{t}}=\mathrm{m}_{\mathrm{m}}$ & $\mathrm{N}$ & $\check{\mathrm{r}}$ & $\mathrm{Q}_{1} \check{\mathrm{r}}$ & $\mathrm{Q}_{2} \check{\mathrm{r}}$ & $\check{\mathrm{s}}$ & $\mathrm{Q}_{1} \check{\mathrm{s}}$ & $\mathrm{Q}_{2} \check{\mathrm{s}}$ & Extinct & $\mathrm{Y}$ \\
\hline 5.5 & 5.5 & 10 & 0.00 & 0.00 & 0.12 & 2.0 & 2.0 & 2.1 & no & - \\
5.5 & 7.0 & 10 & 0.00 & 0.00 & 0.00 & 3.1 & 3.0 & 3.2 & no & - \\
5.5 & 10.0 & 10 & -0.12 & -0.24 & 0.00 & 6.5 & 6.2 & 6.8 & no & - \\
5.5 & 15.0 & 10 & -0.36 & -0.37 & -0.24 & 20.1 & 19.2 & 22.2 & no & - \\
5.5 & 30.0 & 10 & -1.28 & -1.59 & -1.08 & $*$ & $*$ & $*$ & 10 times & 200 \\
5.0 & 7.0 & 10 & 0.50 & 0.49 & 0.53 & 3.5 & 3.5 & 3.6 & no & - \\
5.5 & 7.0 & 10 & 0.00 & 0.00 & 0.00 & 3.1 & 3.0 & 3.2 & no & - \\
6.0 & 7.0 & 10 & -0.33 & -0.44 & 0.00 & 2.5 & 2.5 & 2.8 & 2 times & 451 \\
6.5 & 7.0 & 10 & -0.28 & -0.51 & 0.00 & 2.4 & 2.1 & 2.5 & 10 times & 403 \\
& & & & & & & & & &
\end{tabular}




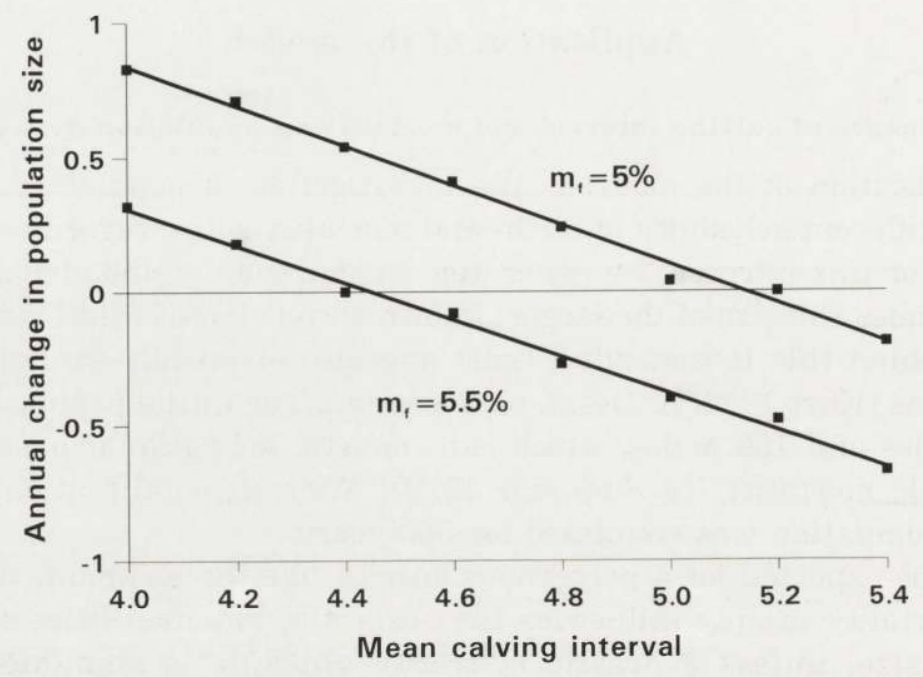

Fig. 3. Relation between calving interval and annual change in population size under different female mortality schedules $\left(\mathrm{m}_{0}=20\right.$ years, $\mathrm{f}_{0}=8$ years, $\Delta_{0}=4$ years, $\left.\mathrm{m}_{\mathrm{m}}=\mathrm{m}_{\mathrm{t}}=7 \%\right)$.

elephant populations in Sri Lanka and southern India, female mortality is thought to range from 5 to $5.5 \%$ (Kurt 1974, Sukumar 1989). To reach a stable population size, this would suggest a mean calving interval of 5.2 years and 4.4-4.5 years, respectively. This is in accordance with values derived from field observations (Kurt 1970, 1974, McKay 1973, Sukumar 1989).

Note that if the minimum female mortality is assumed to be $5 \%$ and the minimum calving interval 4 years, the maximum possible increase of the population size is within $1 \%$ per year (Table 1). This estimate is far below the $4 \%$ maximum possible increase predicted by the African elephant model of Hanks and McIntosh (1973).

\section{The inheritance of the tusk allele}

In southern Sri Lanka, the number of tuskers is relatively high and there are historical records since the 4 th century B.C. The available data suggest a slightly higher mortality for tuskers (about 10\%) than for maknas until the early 16th century (about 7.5\%; Kurt et al. 1995). In the beginning of that period, there were only about 5\% maknas of an estimated total of 450 males, while at the end of that period about $85 \%$ of the males were maknas (Kurt et al. 1995). If we assume the population to have been in Hardy-Weinberg-equilibrium with regard to the tusk allele in the beginning of this period, the estimated frequency of the tusk allele might have been about $80 \%$ in the case of dominance or $95 \%$, if the allele is supposed to be recessive.

If we first omit sexual selection, the results of the simulation suggest the following: If recessivity is assumed, the frequency of the tusk allele decreases from 

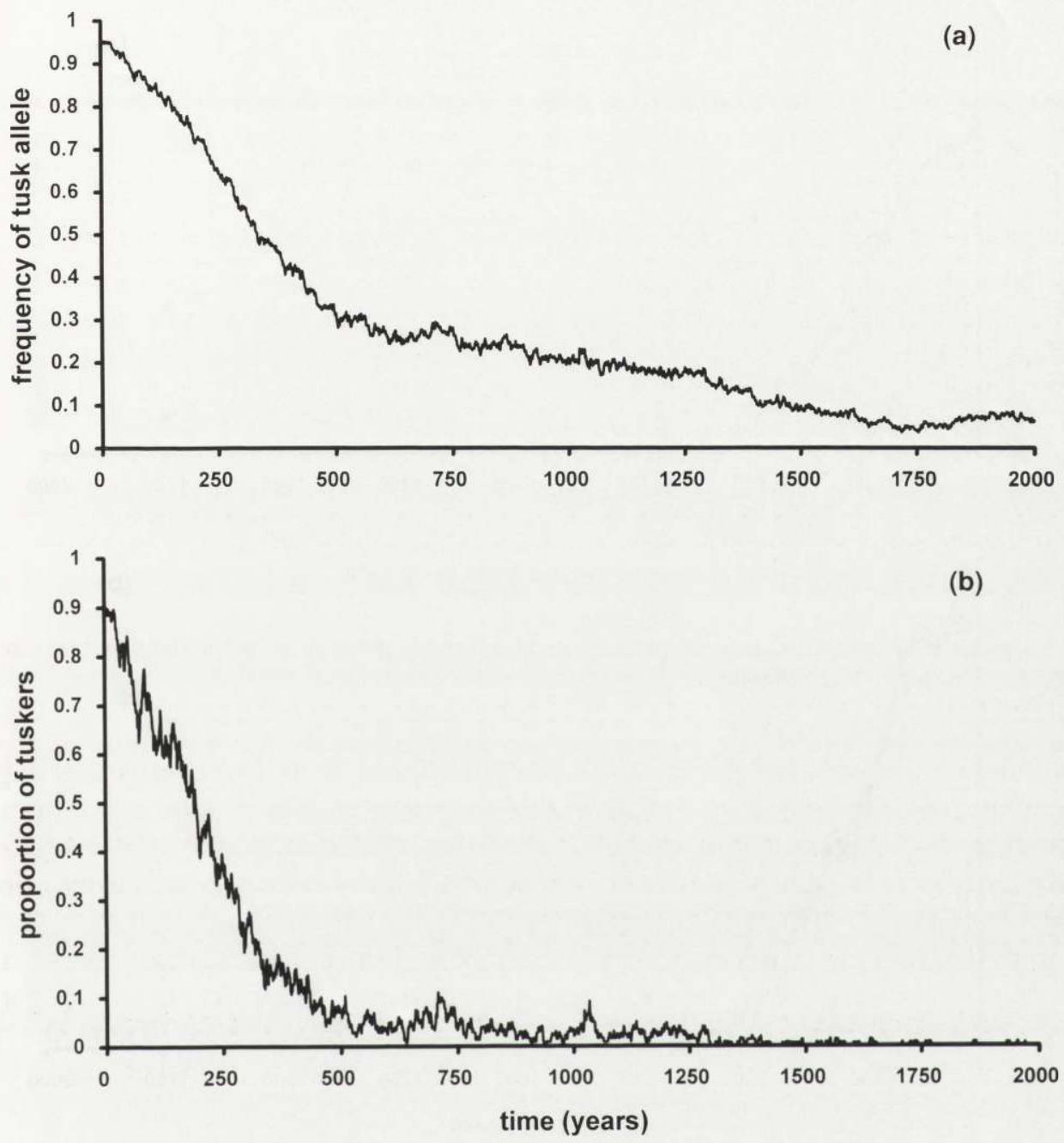

Fig. 4. Frequency $(p)$ of the tusk allele in reproductive adults (a) and proportion of tuskers among reproductive males $(b)$, if the tusk allele is recessive ( $m_{0}=20$ years, $f_{0}=8$ years, $\Delta=4.43$ years, initial $\left.p=95 \%, \mathrm{~m}_{\mathrm{f}}=5.5 \%, \mathrm{~m}_{\mathrm{m}}=7.5 \%, \mathrm{~m}_{\mathrm{t}}=10 \%\right)$. Results of one simulation run as an example.

$95 \%$ to about $25 \%$ within 500 years. After 2000 years, its frequency has lowered to about $5 \%$, and tuskers are very rare in the population (Fig. 4). If the tusk allele is assumed to be dominant, despite its initial frequency of $80 \%$, it will be driven to extinction within about 750 years (Fig. 5). Thus, if tuskers are continuously preferred in hunting or capturing, a dominant inheritance of the tusk allele without sexual selection in favour of phenotypic tuskers will lead to the extinction of the allele. However, this only holds for a starting allele frequency of $80 \%$ or lower. A simulation of dominant inheritance of the tusk allele with an initial 

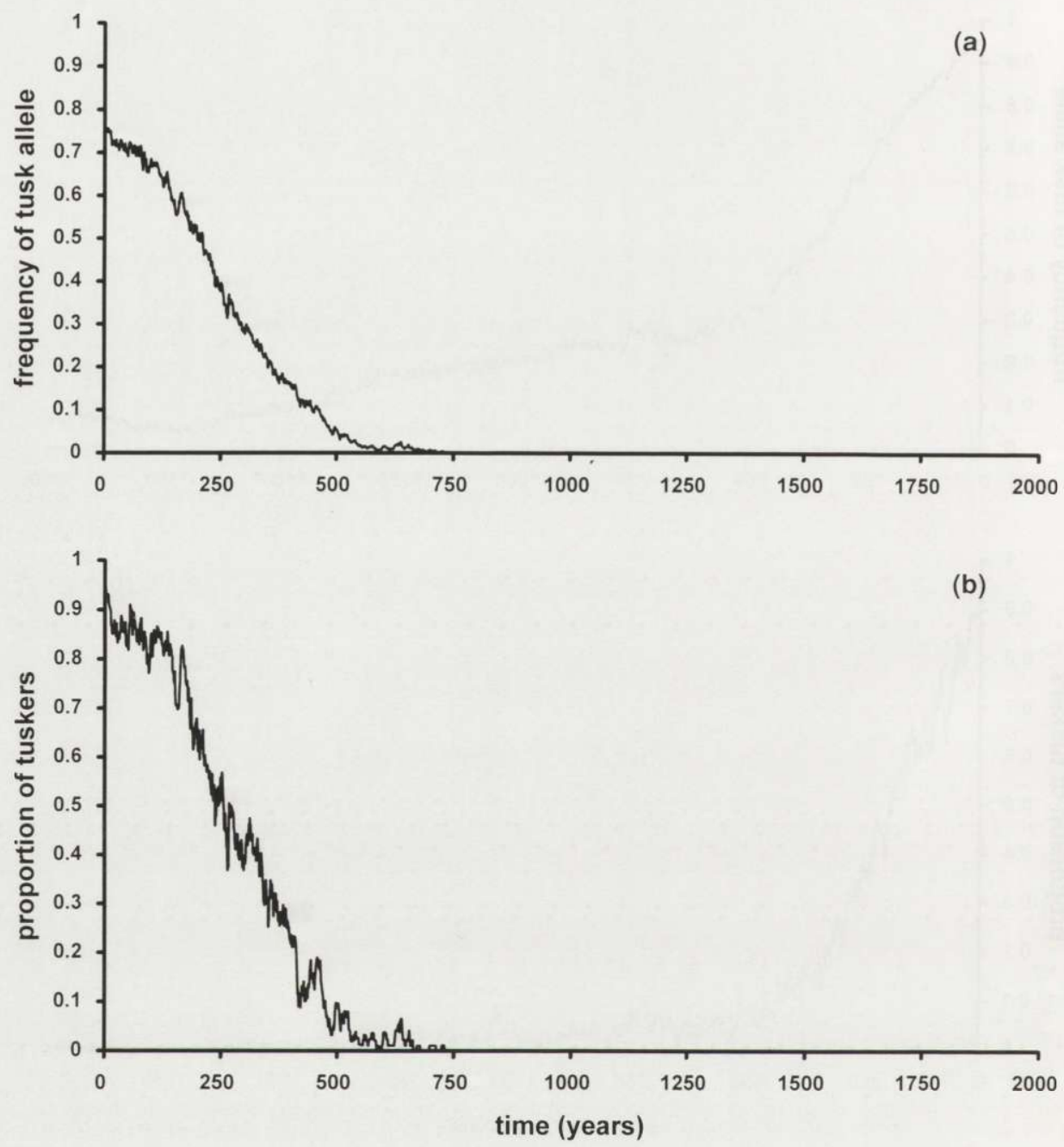

Fig. 5. Frequency $(p)$ of the tusk allele in reproductive adults (a) and proportion of tuskers among reproductive males (b), if the tusk allele is dominant (initial $p=80 \%$; see Fig. 4 for further details).

frequency of 95\% makes the model unpredictable: Even though the tuskers suffer from higher mortality than the maknas, the makna allele becomes extinct in some simulation runs due to random drift (Fig. 6). In other simulations, the frequency of the tusk allele fluctuates between about $85 \%$ and $99 \%$, without any long-term trend (Fig. 6). However, if the frequency of the tusk allele is lowered by random drift to a critical value of about $80 \%$, the lower mortality of maknas immediately leads to a further decrease of the tusk allele, and its extinction is only a matter of several hundred simulated years (Fig. 6). 
(a)

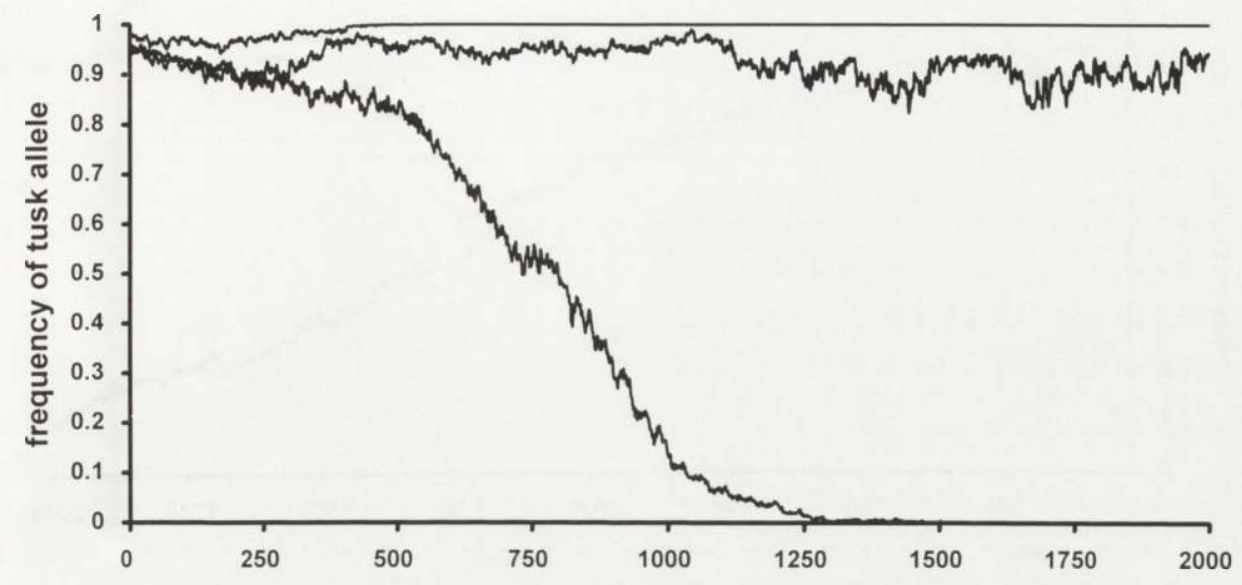

(b)

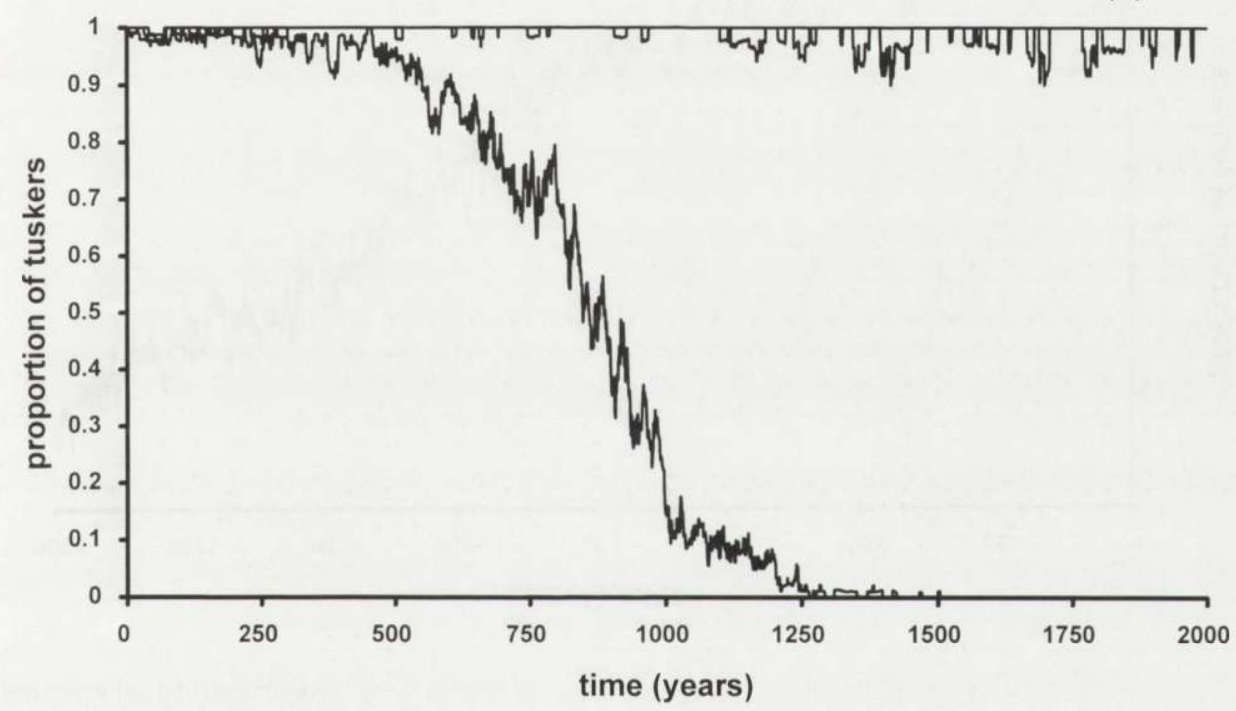

Fig. 6. Three different simulation results for the frequency $(p)$ of the tusk allele in reproductive adults (a) and proportion of tuskers among reproductive males (b), if the tusk allele is dominant (initial $p=95 \%$; see Fig. 4 for further details).

The inclusion of sexual selection in favour of tuskers has a marked influence on the simulation: If we assume tuskers to be twice as successful in mating as maknas $(s=2)$, the lowered mortality of maknas is counteracted, ie there is no significant long-term change in allele frequencies. If the tuskers are assumed to have only a $50 \%$ advantage in mating $(s=1.5)$, within 2000 simulated years the frequency of the tusk allele decreases from $80 \%$ to $10-15 \%$, but it does not become 

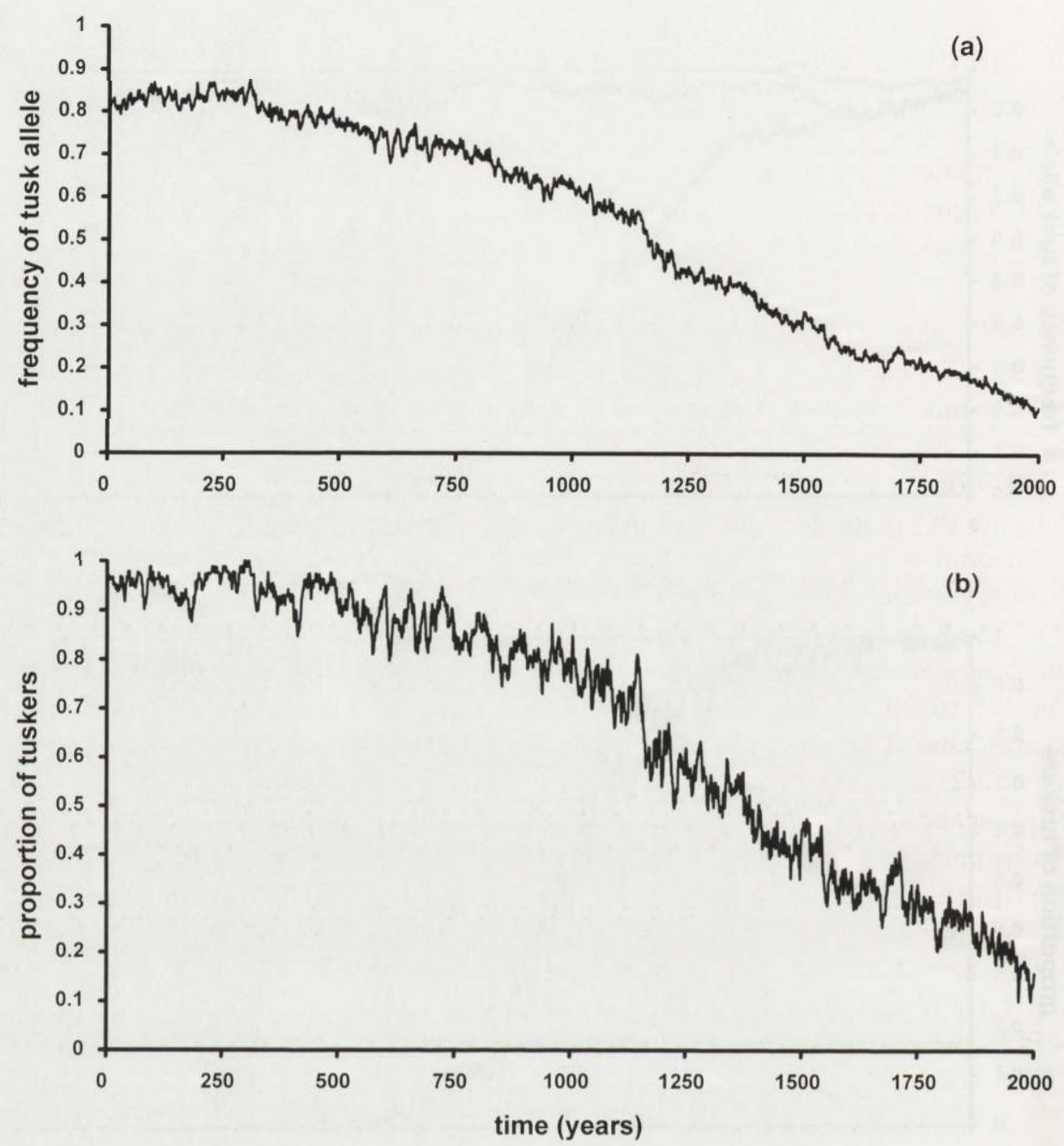

Fig. 7. Frequency $(p)$ of the tusk allele in reproductive adults (a) and proportion of tuskers among reproductive males (b), if the tusk allele is dominant and tuskers have a reproductive advantage of $50 \%$ over maknas (initial $p=80 \%$; see Fig. 4 for further details).

extinct (Fig. 7). Tuskers will be present at a frequency of about $10-15 \%$ after the simulated 2000 years. This figure corresponds with the historical record (cf Kurt et al. 1995). If the selective advantage of tuskers is reduced to $10-30 \%$, the model predicts the extinction of the tusk allele.

The simulation of recessive inheritance of the tusk allele in combination with sexual selection could also explain a decrease in the tusk allele frequency without extinction within 2000 years under the given mortality schedule. However, if we assume that there had been a time with similar mortality rates for all the males, 
before selective hunting and capturing started, the model predicts that, given a reproductive advantage for tuskers, a dominant makna allele would have become extinct.

Consequently, we conclude that under the assumption of an autosomal single gene or major gene inheritance, the tusk allele is most likely dominant, and tuskers probably have a slight reproductive advantage over maknas.

\section{Conclusions}

The robust stochastic model presented in this paper predicts some characteristics of Asian elephant populations, which are corroborated by real data (Kurt et al. 1995). It shows that male mortality is of little influence on population size as long as the sex ratio is not skewed towards females above a critical value. The population dynamics is mainly determined by a specific combination of female age at puberty, mean calving interval, and female mortality.

For the inheritance of the tusk character, the simulations suggest a dominant inheritance of the tusk allele in combination with moderate sexual selection in favour of tuskers. Despite the fact that occasional observations in Sri Lanka indicated that there is no female choice neither in favour of tuskers nor of maknas (Kurt 1992, Ishwaran 1993), it must be considered that in Sri Lanka elephants live in secondary habitats. But in primary habitat, where bark and climbers may be an important part of the diet of solitary adult bulls (Olivier 1978), tuskers may indeed have an ecological and, in consequence, a reproductive advantage. In any case, the prediction of our model is definitely a selective advantage for tuskers, which, however, may be slight enough to be overlooked in field observations. Since the results of the simulations are corroborated by census data (see also Kurt et al. 1995), we conclude that our model may serve as a powerful tool to predict the effect of conservation efforts on populations of the Asian elephant (cf Kurt et al. 1995).

Acknowledgements: We thank the R. \& R. Schlageter-Stiftung (Zoologischer Garten, Zürich) for support. We are indebted to Prof G. B. Hartl for many valuable comments during the realization of the simulation model and for critically reviewing the manuscript.

\section{References}

Digby S. 1971. War-horse and elephant in the Dehli Sultanate, a study of military supplies. Orient Monographs, Oxford: 5-100.

Evans G. H. 1910. Elephants and their diseases. Government Printing, Rangun: 1-343.

Fowler C. W. and Smith T. 1973. Characterizing stable populations: An application to the African elephant population. Journal of Wildlife Management 37: 513-523.

Hanks J. and McIntosh J. E. A. 1973. Population dynamics of the African elephant (Loxodonta africana). Journal of Zoology, London 169: 29-38.

Ishwaran N. 1993. Ecology of the Asian elephant in lowland dry zone habitats of the Mahaweli river basin, Sri Lanka. Journal of Tropical Ecology 9: 169-182. 
Jayewardene J. 1994. The elephant in Sri Lanka. Wildlife Heritage Trust of Sri Lanka, Colombo: $1-128$.

Kurt F. 1970. A comparison of reproduction in tame and wild elephant (Elephas maximus). IUCN XI. Technical meeting 1: 148-154.

Kurt F. 1974. Remarks on the social structure and ecology of the Ceylon elephant in the Yala National Park. [In: The behaviour of ungulates and its relation to management. V. Geist and F. Walther, eds]. IUCN Publications, New Series 24 (1): 618-634.

Kurt F. 1992. Das Elefantenbuch. Rasch und Röhring, Hamburg: 1-239.

Kurt F., Hartl G. B. and Tiedemann R. 1995. Tuskless bulls in Asian elephant Elephas maximus History and population genetics of a man-made phenomenon. [In: Ecological genetics in mammals II. G. B. Hartl and J. Markowski, eds]. Acta Theriologica, Suppl. 3: xx-xx.

McKay G. M. 1973. The ecology and behavior of the Asiatic elephant in southeastern Ceylon. Smithsonion Contributions to Zoology 125: 1-113.

Nair P. G., Radhakrishnan K. and Chandrasekharan 1992. Mating behaviour of the Asian elephant in captivity. [In: The Asian elephant: ecology, biology, diseases and management. E. G. Silas, M. Krishna, G. Nair and G. Nirmalan, eds]. Lumiere Printing Works, Trichur: 38-40.

Olivier R. C. D. 1978. On the ecology of the Asian elephant. Ph D thesis, University of Cambridge, Cambridge: $1-453$.

Poole J. H. 1987. Rutting behaviour in African elephants. Behaviour 102: 283-316.

Poole J. H. 1989. Mate guarding, reproductive success and female choice in African elephants. Animal Behaviour 37: 842-849.

Santiapillai C. and Suprahman H. 1985. Elephants in Indonesia (Sumatra). WWF Monthly Report, December 1985. World Wildlife Fund, Gland, Switzerland: 287-296.

Sivaganesan N. and Sathyanarayana M. C. 1993. Mortalities of trees caused by elephants in Mudumalai wildlife sanctuary, South India. [In: Proceedings of International Seminar on the Conservation of the Asian Elephant. J. C. Daniel, ed]. Bombay Natural History Society, Bombay: $65-72$.

Sukumar R. 1989. The Asian elephant: ecology and management. Cambridge University Press, Cambridge: 1-219.

Sukumar R. 1992. The Asian elephant: ecology and management. Cambridge University Press, Cambridge: $1-255$.

Toke Gale U. 1974. Burmese timber elephants. Trade corporation, Rangoon: 1-152.

Trautmann T. R. 1982. Elephants and the Mauryas. [In: India: History and thought. Mukerjee, ed] Subarnarekha, Calcutta: 245-281.

Wu L. S.-Y. and Botkin D. B. 1980. Of elephants and men: A discrete, stochastic model for long-lived species with complex life histories. American Naturalist 116: 831-849.

Received 7 August 1995, accepted 15 September 1995. 